\title{
Abordaje de la demanda quirúrgica en el este de África. Modelos de cooperación en cirugía. Organización de una campaña sostenible
}

\author{
Silvestra Barrena Blázquez ${ }^{1, *}$ y José María Muguerza Huguet ${ }^{2}$ \\ 1 Graduado en Enfermería, Hospital Universitario Príncipe de Asturias; Máster Universitario en \\ Acción Humanitaria Sanitaria, Universidad de Alcalá \\ 2 Médico especialista del Servicio de Cirugía General y Aparato Digestivo, exprofesor asociado de \\ Cirugía, Facultad de Medicina y Ciencias de la Salud, Universidad de Alcalá \\ * Autor correspondencia: silvebarrena@gmail.com
}

Recibido: 4/10/2019; Aceptado: 5/11/2019; Publicado: 29/11/2019

Resumen: 1) Antecedentes: La atención quirúrgica es parte esencial de la atención de salud y se asocia con una mayor esperanza de vida, sin embargo, muchos países de bajos y medios ingresos no logran alcanzar niveles básicos en este servicio. Las mejoras en la capacidad y la prestación de servicios quirúrgicos deben ser un componente principal del fortalecimiento de los sistemas de salud. 2) Método: Se realiza una búsqueda en PubMed mediante la combinación de palabras claves como "Surgery", "Low and middle income countries", "Health care", "Infrastructure", "Africa", "Cooperation". Se utiliza los registros de los pacientes, screening y procedimientos quirúrgicos, facilitados por el proyecto "Cirugía en Turkana". 3) Resultados: Los procedimientos quirúrgicos están entre las intervenciones de salud más rentables y ha empezado a tomar fuerza el papel de la cirugía como parte de la asistencia sanitaria primaria. Las intervenciones realizadas en la campaña 2018 de "Cirugía en Turkana" fueron patologías de cirugía general (58\%), traumatología (24\%), ginecología $(12 \%)$, cirugía maxilofacial $(6 \%)$ y cirugías de urgencias $(9,5 \%)$. Las patologías más demandadas por especialidades fueron los tumores de partes blandas, fracturas óseas, prolapsos uterinos y labios leporinos. 4) Conclusión: La cirugía es una parte esencial de la atención médica. Las complicaciones son comunes. El quirófano debe ser un entorno de trabajo seguro para los profesionales y para los pacientes. Es importante formar al personal local y concienciar a las autoridades políticas para mejorar las infraestructuras. Los proyectos de cooperación quirúrgicos permiten tratar patologías y disminuir discapacidades.

Palabras Clave: Cirugía, Atención sanitaria, Países de baja y media renta, Cooperación, Infraestructuras, Procedimientos quirúrgicos, Africa, Morbilidad, Mortalidad, Campañas quirúrgicas.

Abstract: 1) History: Surgical care is an essential part of health care and is associated with a longer
life expectancy, however, many countries of low and middle income fail to reach basic levels in this
service. Improvements in the capacity and provision of surgical services should be a major
component of the strengthening of health systems.2) Method: A PubMed search is carried out
through the combination of keywords such as "Surgery", "Low and middle income countries",
"Health care", "Infrastructure", "Africa", "Cooperation". Patient records, screening and surgical
procedures are used, facilitated by the Surgery project in Turkana. 3) Results: Surgical procedures
are among the most cost-effective health interventions and the role of surgery as part of primary
health care has begun to take hold. The interventions carried out in the 2018 campaign of Surgery
in Turkana were pathologies of general surgery ( $58 \%)$, traumatology $(24 \%)$, gynecology (12\%),
maxillo facial surgery (6\%) and emergency surgeries $(9.5 \%)$. The most demanded pathologies by
specialties were soft tissue tumors, bone fractures, uterine prolapses and cleft lips. 4) Conclusion:
Surgery is an essential part of medical care. Complications are common. The operating room should 
be a safe work environment for professionals and patients. It is important to train local staff and raise awareness among political authorities to improve infrastructure. Surgical cooperation projects allow to treat pathologies and reduce disabilities.

Key words: Surgery, Health Care, Countries of low and middle income, Cooperation, Infrastructure, Surgical procedures, Africa, Morbidity, Mortality, Surgical campaigns.

\section{Introducción}

La salud es un derecho universal que se entiende en un sentido amplio, es decir, no solo es la salud física, sino que incluye los determinantes de salud. Cuando hablamos de determinantes sociales de salud tenemos en cuenta las circunstancias en que las personas nacen, crecen, viven, trabajan y envejecen, incluido el sistema de salud. Esas circunstancias son el resultado de la distribución del dinero, el poder y los recursos, tanto a nivel mundial, nacional y local. La principal estrategia para abordar las desigualdades en salud es avanzar hacia la Cobertura Universal de calidad, integral y servicios de salud centrados en las personas. Muchos de los países de la Región Africana de la OMS no alcanzarán los Objetivos de Desarrollo del Milenio (ODM) y para alcanzar el Objetivo de Desarrollo Sostenible 3 (ODS) sobre: "Garantizar una vida sana y promover el bienestar para todos en todas las edades" la mayoría de los países de Africa tendrán que hacer frente a las deficiencias de los sistemas de salud y por lo tanto a los determinantes sociales de salud.

En África solo hay 2 médicos por cada 10.000 habitantes, mientras en Europa hay 33 por cada 10.000. A nivel mundial hay 29 enfermeros y matronas por cada 10.000 habitantes, pero solo 12 por cada 10.000 en África y 80 por cada 10.000 en Europa [1]. Los países de bajos y medianos ingresos (PIBM), que representan el $48 \%$ de la población mundial, solo tienen el 20\% de especialistas, o el 19\% de todos los cirujanos, el 15\% de los anestesiólogos y el 29\% de los obstetras [2].

La forma de fortalecer y acelerar la sanidad en los PIBM es mediante la cooperación sur-sur y triangular. La OMS está cambiando de perspectiva y reorientando sus recursos, intensificando su colaboración y su apoyo con aquellos gobiernos e instituciones locales, sector privado y sociedad civil que contribuyen a mejorar la acción sanitaria. Intensificar la colaboración con los países más pobres es fundamental para luchar por la equidad en salud [3].

El proyecto de "Cirugía en Turkana" realiza campañas quirúrgicas anuales en la región de Turkana en Kenia, para prestar tratamiento quirúrgico a una población que no tiene acceso a ello. Este modelo de cooperación va a ser el objeto de análisis de este artículo.

\subsection{Objetivos}

1. Revisar la situación sanitaria especializada en los países del este de África y las dificultades en el acceso a la atención quirúrgica.

2. Analizar los datos obtenidos en la campaña del 2018 del proyecto Cirugía en Turkana en Kenia.

\section{Material y métodos}

\subsection{Material}

Se realizó una búsqueda en la base de datos PubMed y EMBASE, y en los accesos web de los países de la zona combinando palabras clave como "Cirugía"," acceso"," Atención Sanitaria", "Países de baja y media renta", "Cooperación", "Infraestructuras", "Carga global de enfermedad", "Procedimientos quirúrgicos", "África", "Años de vida y discapacidad", "Personal sanitario", "Cirugía de emergencia", "Morbilidad", "Mortalidad", "Esperanza de vida", "Desigualdad", "Campañas quirúrgicas"," Cirugía en Turkana", "Kenia", "Screening". Se han incluido aquellos artículos que se ajustaban a los términos anteriormente citados. 


\subsection{Métodos}

Como modelo de actuación quirúrgica sobre el terreno se toma la Campaña de Cirugía en Turkana 2018 que selecciona las necesidades quirúrgicas basadas en criterio de diagnóstico clínico. Se realiza un primer screening llevado a cabo por personal sanitario local, durante unos meses antes de comenzar la campaña quirúrgica. Posteriormente, se realiza un segundo screening con pruebas complementarias como ecografía, radiología simple y uso de la telemedicina, llevado a cabo durante la campaña quirúrgica por personal del equipo.

\section{Resultados}

Cirugía en Turkana es un proyecto de cooperación, que presta asistencia quirúrgica a la población de Turkana en el Hospital del Distrito, en Lodwar. El hospital no cuenta con el número suficiente de cirujanos que atienda a esta gran región de Kenia. Este proyecto proporciona material y medios de exploración para las cirugías, comisiones quirúrgicas formadas por cirujanos, ginecólogos, traumatólogos, maxilofacial, enfermeras y anestesistas, y formación del personal sanitario local para que puedan atender las necesidades quirúrgicas de la población el resto del año.

En la campaña 2018 en el hospital de Lodwar se atendieron en consulta a 505 pacientes (70\%), se operaron a $190(27 \%)$, y se derivaron a 22 (3\%) a otros hospitales.

Tabla I Clasificación de intervenciones por especialidades quirúrgicas en la campaña de “Cirugía en Turkana” 2018.

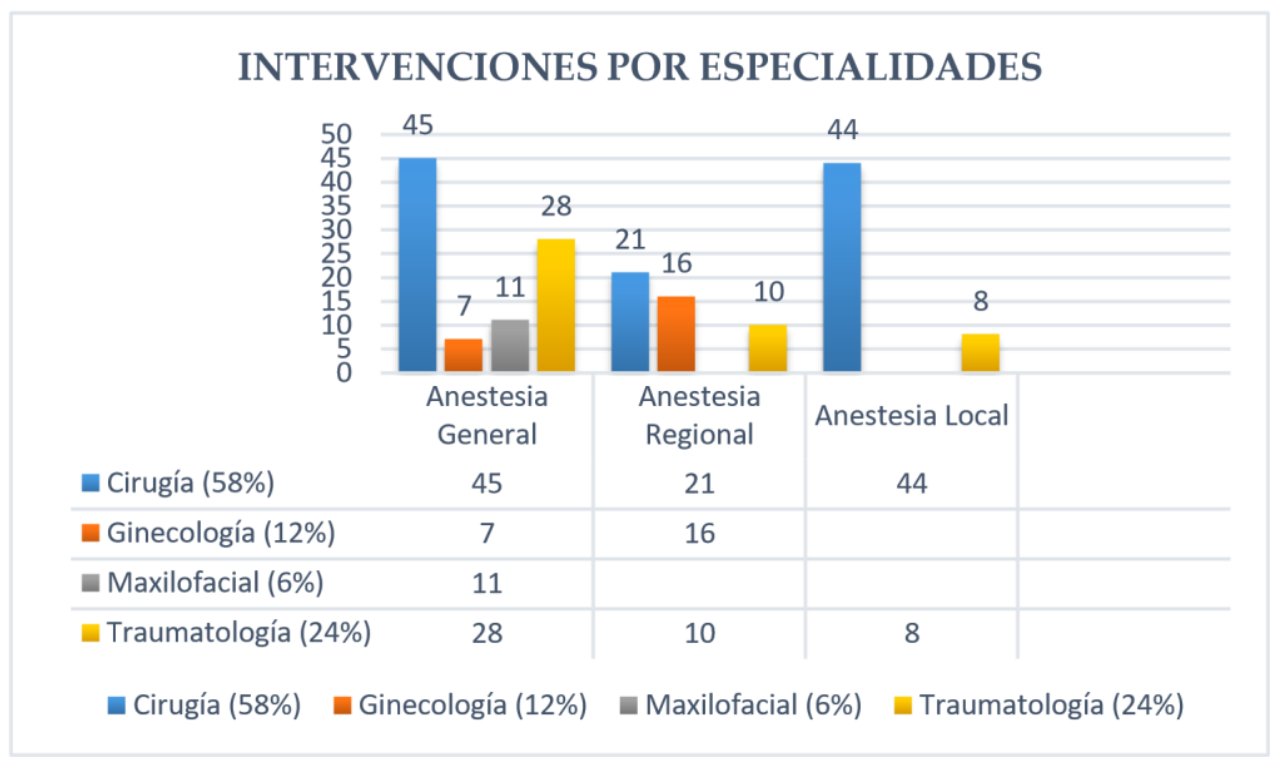

Se llevaron a cabo 18 intervenciones de forma urgente (9,5\% del total) con el diagnóstico de: cesáreas (7), embarazos ectópicos (2), desgarros perineales tras parto (3), politraumas (2), obstrucciones intestinales (2) y hematomas intracraneales (2).

Entre las intervenciones programadas de cirugía maxilo facial destacaron en número los pacientes por labios leporinos entre siete y veinte meses. En la especialidad de ginecología se llevaron a cabo, entre otras, cinco intervenciones de histerectomía vaginal en pacientes con prolapsos uterinos y tres mastectomías, una bilateral por ginecomastia bilateral y dos por adenocarcinoma de mama. En la especialidad de traumatología se operaron sobre todo a pacientes con fracturas, tumoraciones de partes blandas y madura foot. En cirugía general se intervinieron a pacientes diagnosticados de hernia inguinal, hidroceles, lipomas, quistes, quemados, etc. 


\section{Discusión}

\subsection{Morbimortalidad en el Este de África}

Las enfermedades transmisibles continúan siendo las principales causas de muerte en la región de África, donde también son las causas y las consecuencias de la pobreza, además del VIH / SIDA, la tuberculosis y la malaria. También la mortalidad materna y neonatal son persistentemente alta y ha aumentado la incidencia de enfermedades no transmisibles como el cáncer, la hipertensión, las enfermedades cardiovasculares, la diabetes, las enfermedades relacionadas con el tabaco, el abuso de drogas y las enfermedades neuropsiquiátricas [4].

Las diferencias en la mortalidad muestran la importancia de los determinantes sociales, generan inquietudes sobre la calidad de los servicios de salud y ofrecen información a los responsables políticos para abordar las desigualdades [5].

La percepción de que la cirugía es una modalidad médica costosa con un bajo coste efectividad ha ido cambiando con el paso del tiempo, imponiéndose la evidencia de que los procedimientos quirúrgicos esenciales están entre las intervenciones de salud más rentables y ha empezado a tomar fuerza el papel de la cirugía como parte de la asistencia sanitaria primaria. Cada vez más, se está demostrando que los servicios hospitalarios básicos pueden ser rentable y no requerir más gastos que procedimientos preventivos como la vacunación contra el sarampión o el tétanos. Así pues, operar a las víctimas de lesiones, tanto para reducir la mortalidad como discapacidades, complicaciones obstétricas y emergencias quirúrgicas neonatales, patología abdominal de emergencia y procedimientos simples como hernias, hidroceles y cataratas, están adquiriendo más peso en la salud pública y debería financiarse adecuadamente para conseguir la cobertura universal de salud.

Según fuentes del gobierno de Turkana la tasa de mortalidad materna es de 1.594 bebés por cada 1.000 y la mortalidad de menores de cinco años es de 74/1.000. La prevalencia del VIH es del 7,6\%. La tasa de vacunación en menores de un año es del $70 \%$. Los niños menores de cinco años con crecimiento retardado son del $24 \%$, y el uso de productos básicos en planificación familiar es del 6,2\%. Las enfermedades más prevalentes en la zona son la malaria, diarrea y enfermedades de la piel. Las patologías más frecuentes en los niños son: anemia, marasmo (desnutrición por déficit energético), Kwashiorkor (desnutrición por déficit de proteínas en la alimentación), infección ocular y neumonía.

\subsection{Acceso a la atención sanitaria en los países de ingresos bajos y medianos}

El acceso adecuado a la atención de emergencia reduce sustancialmente la mortalidad. En África el acceso físico a la atención hospitalaria es deficiente, con marcadas inequidades dentro de los países y entre ellos. La atención quirúrgica de emergencia se refiere a la capacidad del sistema de salud de ofrecer servicios curativos para patologías que amenazan la vida. Mejorar la calidad y el acceso a estos servicios quirúrgicos de emergencia podrían reducir el $45 \%$ de la mortalidad y el 36\% de las discapacidades en PIBM [6].

En los hospitales de primer nivel en PIBM no hay personal especializado en cirugía ni en anestesia. Los procedimientos mayores son derivados a hospitales de segundo o tercer nivel con el agravante que suelen estar lejos de las poblaciones más desfavorecidas. En África el 29\% de la población total y el $28 \%$ de las mujeres en edad fértil se encuentran geográficamente marginadas de la atención médica, obstétrica y quirúrgica de emergencia. Las zonas rurales donde viven suelen estar a más de 2 horas de viaje del hospital público más cercano, las malas carreteras y la falta de transporte adecuado constituyen, entre otras, barreras para acceder a los servicios quirúrgicos.

\subsection{Desafíos de la cirugía en los países de ingresos bajos y medianos}

Se ha comprobado que la atención quirúrgica esencial es indispensable en la atención de salud de la población y está vinculada con una mayor esperanza de vida y disminución de la morbimortalidad. La falta de recursos humanos y materiales, junto con una infraestructura deficiente, son consecuencia de que no se proporcione una cirugía adecuada y segura en muchos países de África 
que disponen de menos de 2 quirófanos por cada 100.000 habitantes frente a 14 por cada 100.000 habitantes que dispondrían en los países de altos ingresos [7].

El África subsahariana tiene el $11 \%$ de la población mundial y el $24 \%$ de la carga global de morbilidad y tiene menos del $1 \%$ del número de cirujanos que tiene Estados Unidos. En 19 de los 52 países de África Subsahariana menos del $50 \%$ de los partos fueron atendidos por parteras cualificadas. Según la OMS para alcanzar el quinto Objetivo de los ODM de "reducir la mortalidad materna en tres cuartas partes" el $80 \%$ de los partos deberán ser atendidos por personal sanitario especializado. La escasez de profesionales capacitados es debido a una serie de causas como el bajo número de graduados en medicina, bajos salarios, incapacidad de motivar a los profesionales para que trabajen en zonas rurales, falta de compromiso de los gobiernos por aumentar los recursos humanos en los servicios de salud y el crecimiento de la población. Todo esto representa para estos países una lucha para alcanzar los ODM [8].

Tabla II Recomendaciones de la OMS. Distribución del personal sanitario en distintas regiones por cada 10.000 habitantes [9].

\begin{tabular}{l|cc|}
\hline Recomendado por OMS & \multicolumn{2}{|c|}{ Personal sanitario/Habitantes } \\
\hline \multicolumn{1}{|c|}{ Mundo } & $23 / 10.000$ \\
\hline Región & Medico/Habitantes & $1,92 / 10.000$ \\
Turkana & $0,14 / 10.000$ & $11 / 10.000$ \\
Africa & $2 / 10.000$ & $79 / 10.000$ \\
Europa & $32 / 10.000$ & Enfermero/Habitantes \\
\hline
\end{tabular}

\subsection{Infraestructura limitada y falta de atención quirúrgica}

La atención de emergencia extrahospitalaria (OHEC) ha demostrado que reduce la morbilidad y la mortalidad para enfermedades graves, por eso es importante disponer de transporte adecuado, ambulancias para trasladar a los enfermos y mejorar el acceso de los pacientes a la atención prehospitalaria [10]. Un estudio realizado en hospitales de primer nivel de 5 países subsaharianos demostró que menos de la mitad de los hospitales estudiados tenían la capacidad de ofrecer atención de emergencia las 24 horas, y menos del $65 \%$ disponían de suministros de agua y electricidad [11]. Hay países que han asociado el objetivo de la cobertura universal de salud a reformas en la salud como China y Estonia, mientras que otros países están desarrollando políticas que se centran en los ODM y expandiendo estrategias para mejorar el acceso a servicios y disponer de protección financiera como es el caso de Etiopía y Tanzania [12].

La sanidad es uno de los sectores menos desarrollados del Condado de Turkana, entre las instalaciones de salud cuenta con 12 hospitales, 13 centros de salud, 168 dispensarios, 26 clínicas y un total de 800 trabajadores de salud en todo el territorio. En muchas áreas de la población la distancia hasta un establecimiento sanitario es de $20 \mathrm{Km}$, con una red de carreteras inexistentes.

\subsection{Papel de la cooperación internacional en las necesidades quirúrgicas}

La principal función que tienen los modelos de cooperación internacional es hacer efectivo el derecho a la salud para todas las personas, especialmente para aquellas que viven en situación de pobreza, inequidad de género y exclusión social. La cooperación quirúrgica internacional ofrece asistencia sanitaria especializada a una población que habitualmente no tendría acceso si dependiera de los servicios que les proporciona su país, por lo tanto, constituyen un suplemento a los servicios básicos de salud de los PIBM.

Las acciones de las ONG pueden ser temporales o permanentes. Dentro de las plataformas temporales se encuentran las campañas quirúrgicas. No se pretende crear sistemas de asistencia 
paralelos, sino lograr un entorno de estrecha colaboración entre el personal sanitario local y el personal desplazado [13]. El trabajo de las ONG debe plantearse de manera integral, que afecte no solo al hospital donde trabajamos sino también a las condiciones en las que viven los pacientes, involucrar a las organizaciones que trabajan en la comunidad relacionadas con la salud medioambiental, trabajar con la comunidad e instituciones locales con el fin de que los esfuerzos humanitarios de estas campañas quirúrgicas sean sostenibles a largo plazo.

\subsection{La cooperación desde la perspectiva de la enfermera cooperante}

En el hospital de Lodwar, los quirófanos carecen de espacio físico para llevar a cabo las intervenciones quirúrgicas de forma segura; la limpieza y la higiene en general rallan la frontera de lo inadmisible en cualquier otro hospital de un país desarrollado. Debemos prestar asistencia quirúrgica y sanitaria de calidad y para ello debemos aplicar normas y principios de asepsia y antisepsia en el quirófano, para evitar que los pacientes que ingresan para procedimientos quirúrgicos adquieran infecciones intraoperatorias que aumenten la morbilidad y mortalidad de estos. Sería interesante implantar protocolos o fichas para la limpieza del medio hospitalario, y del quirófano concretamente, donde se describan los objetivos de la limpieza, técnicas generales, productos y utensilios, etc., haciendo entender al personal local que allí trabaja, la importancia que tiene la limpieza en la prevención de las infecciones nosocomiales y sin olvidarnos de los medios de los que disponen, sacar el mejor provecho.

Es fundamental conocer el contexto de actuación, identificar y respetar su cultura, normas, valores, creencia, etc., antes de llevar a cabo ningún tipo de intervención y tener presente que el personal local (médicos, enfermeras y medical officers), están en disposición y tienen las competencias necesarias para ofrecer una adecuada atención sanitaria. Las barreras culturales, como la vergüenza y el miedo a la cirugía son un impedimento para que la población entre en contacto con los servicios sanitarios, por eso es necesario concienciar a la población, y hacer partícipe a la comunidad local para comprender y aceptar los beneficios de una intervención quirúrgica temprana [14].

Es necesaria la educación para la salud en las poblaciones más vulnerables, la promoción de buenas prácticas de higiene no solo para evitar la transmisión de enfermedades, sino también para que el postoperatorio de la cirugía evolucione adecuadamente, así como la seguridad alimentaria, rehabilitación, atención y recuperación. Para que esto se lleve a cabo, es preciso una acción integral en salud que valore desde las condiciones en las que vive la población hasta la infraestructura de servicios sanitarios, involucrando y coordinándose con organizaciones que trabajen en la zona en salud medioambiental, sectores sociales y servicios de salud locales.

\subsection{La campaña quirúrgica "Cirugía en Turkana" 2018}

Como hemos podido concluir los tumores de partes blandas (queloides, escarificaciones, lipomas, etc.), y hernias congénitas asociadas a hidroceles, son la patología más frecuente que presentan estos pacientes. Normalmente en los países desarrollados, los pacientes con hernias congénitas son intervenidos después de los 18 meses de edad si no se resuelven de forma espontánea, pero en Turkana se ven pacientes entre 20 y 30 años con este tipo de patología en un estado muy avanzado.

En el oeste de Kenia se realizan una alta tasa de partos en los domicilios por parteras no cualificadas lo que supone un importante problema de salud por la alta tasa de mortalidad materna y neonatal. Hay un gran número de mujeres embarazadas con complicaciones obstétrica que no son enviadas a los servicios de salud para que sean atendidas por profesionales en ginecología y tocología [15]. Por eso, es importante fomentar entre las parteras la necesidad de remitir a las embarazadas a los centros de salud más próximos para el seguimiento de salud materno infantil e incentivar a los responsables políticos de los PIBM a reeducar y definir las funciones de las parteras tradicionales. Durante la campaña de "Cirugía en Turkana 2018" pudimos comprobar que la patología ginecológica más demandada fueron las cesáreas urgentes. 
Según los datos de las operaciones que este año ha realizado "Cirugía en Turkana", podemos ver que la necesidad insatisfecha de atención de labio leporino y/o paladar hendido es significativa en los PIMB. Según los datos de los programas quirúrgicos Operation Smile, OMS, y el Banco Mundial se comprobó que la incidencia de labio leporino sin operar es 2,5 por 100.000 habitantes en Rumania a 28,5 por 100.000 en Camboya [16].

África es una de las regiones donde hay más casos de quemaduras graves en niños por falta de desarrollo y pobreza en sus viviendas, representando una alta tasa de morbilidad y mortalidad. Según un estudio basado en las publicaciones sobre quemados en los países subsaharianos, el $80 \%$ de las quemaduras se dan en niños menores de 10 años representando las escaldaduras el $59 \%$ de las causas y las quemaduras por llama el 33\% [17].

Otra patología frecuente en Turkana es el micetoma o "madura", enfermedad infecciosa local, crónica y progresiva de la piel, tejido subcutáneo y hueso. Comienza con un traumatismo por donde se introduce el agente causal que suelen ser bacterias (actinomicetoma) que se encuentra con más frecuencia en América, u hongos (eumicetoma), que es más frecuente en África por el contacto con espinas de las acacias. Posteriormente se formará un nódulo, que crece de forma gradual hasta convertirse en una masa purulenta. Es una lesión mutilante que puede terminar en una amputación del miembro si no se trata a tiempo. Aunque la parte más afectada suelen ser los pies, también se han visto en hombros, nalgas y espalda [18].

\subsection{La telemedicina es una pieza clave en el futuro de la salud}

Con la ayuda de la telemedicina podemos luchar contra las barreras geográficas aumentando la accesibilidad a los cuidados de salud. Se integró en el proyecto de "Cirugía en Turkana" la telemedicina mediante la cual podemos compartir a tiempo real con un médico especialista a distancia, sugerencias o impresiones sobre el diagnóstico de estos pacientes que a veces son muy útiles no solo por la falta de medios en el hospital de Lodwar para el diagnóstico clínico, sino también por la necesidad de consultar con otros especialistas que no están en terreno.

\subsection{Trabajar juntamente con las autoridades y personal sanitario local}

Es muy importante que la población y las autoridades confíen en los proyectos de cooperación, en su utilidad y resultados con las personas más desfavorecidas. Las autoridades locales tienen que difundir las ventajas que los proyectos quirúrgicos tienen para esa población sin acceso a la sanidad, porque no solo se les curan las patologías, sino que pueden mejorar su economía ya que le será más fácil la incorporación al mercado laboral. Siempre hay que tener presente el valor de la coordinación y comunicación entre los desplazados y el personal local para que nuestro trabajo se continúe haciendo de forma autónoma cuando la campaña haya terminado. También hay que promover un rol activo tanto del personal como de las infraestructuras del hospital para conseguir unos resultados que esa población pueda mantener.

\section{Conclusiones}

1. Disponer de un sistema sanitario justo y equitativo con acceso a la asistencia quirúrgica reduciría la mortalidad y discapacidades de muchas personas víctimas de lesiones y evitaríamos complicaciones obstétricas y emergencias quirúrgicas neonatales. También disminuiría la morbilidad de la patología abdominal de emergencia y procedimientos simples como hernias, cataratas, hidroceles, etc., en PIBM. Invertir en cirugía y anestesia evitaría el 3,5\% de las muertes totales globales.

2. La falta de recursos humanos y materiales y una infraestructura deficiente de los servicios sanitarios son la consecuencia de que no se proporcione una cirugía adecuada y segura en muchos países de África. Para garantizar el derecho a la salud y el acceso a los servicios de calidad necesitamos personal especializado en cirugía y anestesia tanto en los hospitales de 
primer, segundo y tercer nivel y que tengan acceso a ello toda la población incluida la más vulnerable. En los PIBM las campañas quirúrgicas de corta duración son un suplemento en la asistencia quirúrgica, no solo por la atención directa sobre los pacientes sino también por el entrenamiento y aprendizaje de técnicas quirúrgicas por el personal sanitario local.

3. El proyecto de cooperación internacional "Cirugía en Turkana" durante la campaña del 2018, utilizando como medio de diagnóstico principalmente la exploración física llevó a cabo intervenciones de cirugía general, traumatología, ginecológicas y cirugía maxilofacial.

Agradecimientos: Agradezco a mi tutor y compañero el Dr. Mugüerza, el tiempo que ha dedicado a ayudarme con el trabajo dándome su apoyo y buenos consejos. También quiero agradecer al equipo de "Cirugía en Turkana" la oportunidad de haber estado en primera persona en la campaña quirúrgica del 2018 en Lodwar, así como la cesión de material fotográfico aportada por Gisela Fernández-Pretel, fotógrafa del proyecto "Cirugía en Turkana".

Contribución de los autores: Para escribir este artículo de investigación participé en primera persona en la campaña quirúrgica que "Cirugía en Turkana "llevó a cabo en Lodwar en el año 2018.

Conflictos de Intereses: El autor no declara conflicto de intereses. Los fundadores de "Cirugía en Turkana" no tienen ningún papel en el diseño del estudio; en la colección, análisis o interpretación de los datos, en la escritura del manuscrito y en la decisión de publicar los resultados.

\section{Abreviaturas}

Las siguientes abreviaturas son usadas en este manuscrito:

COSECSA: Colegio de Cirujanos de África Oriental, Central y Meridional.

GIEESC: Iniciativa Global para Atención Quirúrgica Esencial y de Emergencia.

ODM: Objetivos de Desarrollo del Milenio.

ODS: Objetivo de Desarrollo Sostenible.

OHEC: Atención de emergencia extrahospitalaria.

OMS: Organización Mundial de la Salud.

ONG: Organización No Gubernamental.

PÁG.: Página.

PIBM: Países de ingresos bajos y medianos.

SSCL: Lista de control de seguridad quirúrgica.

VHC. Virus de la hepatitis C.

VIH: Virus de inmunodeficiencia humana.

\section{Referencias Bibliográficas}

1. World Health Organization. Ginebra, Suiza. World Health Stadistics 2014. Part III. Global health indicators. 6: Health Systems; 128-141. Disponible http://www.who.int/gho/publications/world_health_statistics/2014/es/ Acceso 18 de octubre, 2018.

2. Holmer H, Lantz A, Kunjumen T, Finlayson S, Hoyler M, Siyam A, et al. Global distribution of surgeons, anaesthesiologists, and obstetricians. Lancet Glob Health 2015; 3 Suppl 2: S9-11.

3. The National Coalition on Health Care, The Institute for Healthcare Improvement. Accelerating Change Today: Curing the system: stories of change in chronic illness care. 2002;Disponible: http://www.improvingchroniccare.org/downloads/act_report_may_2002_curing_the_system.pdf. Acceso 2 de noviembre, 2018

4. World Health Organization. Regional Office for Africa. Health in Africa in 2000-2001: a balance sheet. Disponible en: https://afro.who.int/news/health-africa-2000-2001-balance-sheet. Acceso 30 de octubre, 2018 5. Pillay-van Wyk V, Msemburi W, Laubscher R, Dorrington RE, Groenewald P, Glass T, et al. Mortality 
trends and differentials in South Africa from 1997 to 2012: second National Burden of Disease Study. Lancet Glob Health 2016 ;4(9):e642-53.

5. Ouma PO, Maina J, Thuranira PN, Macharia PM, Alegana VA, English M, et al. Access to emergency hospital care provided by the public sector in sub-Saharan Africa in 2015: a geocoded inventory and spatial analysis. Lancet Glob Health 2018 ;6(3): e342-e350.

6. Funk LM, Weiser TG, Berry WR, Lipsitz SR, Merry AF, Enright AC, et al. Global operating theatre distribution and pulse oximetry supply: an estimation from reported data. Lancet $2010 ; 376(9746)$ :10551061.

7. Bergstrom S, McPake B, Pereira C, Dovlo D. Workforce Innovations to Expand the Capacity for Surgical Services. En: Debas HT, Donkor P, Gawande A, Jamison DT, Kruk ME, Mock CN, editors. Essential Surgery: Disease Control Priorities, Third Edition (Volume 1) Washington (DC): International Bank for Reconstruction and Development / The World Bank; 2015.

8. Cirugía en Turkana. Disponible en: http://www.cirugiaenturkana.com/ Acceso 30 de octubre, 2018

9. Kironji AG, Hodkinson P, de Ramirez SS, Anest T, Wallis L, Razzak J, et al. Identifying barriers for out of hospital emergency care in low and low-middle income countries: a systematic review. BMC Health Serv Res 2018 ;18(1):291-018-3091-0.

10. Hsia RY, Mbembati NA, Macfarlane S, Kruk ME. Access to emergency and surgical care in subSaharan Africa: the infrastructure gap. Health Policy Plan 2012;27(3):234-244.

11. Boerma T, Eozenou P, Evans D, Evans T, Kieny MP, Wagstaff A. Monitoring progress towards universal health coverage at country and global levels. PLoS Med 2014;11(9): e1001731.

12. Corlew DS. Economic modeling of surgical disease: a measure of public health interventions.

13. World J Surg $2013 ; 37(7): 1478-1485$.

14. Briesen S, Geneau R, Roberts H, Opiyo J, Courtright P. Understanding why patients with cataract refuse free surgery: the influence of rumours in Kenya. Trop Med Int Health 2010;15(5):534-539.

15. Bucher S, Konana O, Liechty E, Garces A, Gisore P, Marete I, et al. Self-reported practices among traditional birth attendants surveyed in western Kenya: a descriptive study. BMC Pregnancy Childbirth 2016;16(1):219-016-1007-8.

16. Stewart BT, Carlson L, Hatcher KW, Sengupta A, Vander Burg R. Estimate of Unmet Need for Cleft Lip and/or Palate Surgery in india. JAMA Facial Plast Surg 2016;18(5):354-361.

17. Nthumba PM. Burns in sub-Saharan Africa: A review. Burns 2016;42(2):258-266.

18. Van de Sande WW. Global Burden of human mycetoma: a systematic review and meta-analysis. PLoS Negl Trop Dis 2013;7(11): e2550. 